PROCEEDINGS OF THE

AMERICAN MATHEMATICAL SOCIETY

Volume 127, Number 5, Pages 1453-1462

S 0002-9939(99)05046-7

Article electronically published on January 29, 1999

\title{
ON THE STRUCTURE OF THE SET OF BOUNDED SOLUTIONS ON A PERIODIC LIÉNARD EQUATION
}

\author{
JUAN CAMPOS AND PEDRO J. TORRES \\ (Communicated by Hal L. Smith)
}

\begin{abstract}
We describe the dynamics of a class of second order periodic differential equations whose main feature is a monotone nonlinearity. It is proved that the set of bounded solutions is homeomorphic to the graph of a decreasing function.
\end{abstract}

\section{INTRODUCTION}

In this paper, we are concerned with the periodic Liénard equation

$$
x^{\prime \prime}+f(x) x^{\prime}+g(x)=p(t),
$$

where $f, g:(a, b) \rightarrow \mathbb{R},-\infty \leq a<b \leq+\infty$ are locally Lipschitz continuous functions. Throughout the paper, we assume the following main assumptions:

i) $g$ is strictly decreasing.

ii) $f(x) \geq 0$ for all $x \in(a, b)$.

iii) The function $p: \mathbb{R} \rightarrow \mathbb{R}$ is continuous and periodic with minimal period $T>0$.

We are interested especially in the case where $(a, b) \neq \mathbb{R}$, since our original intention was to consider singular nonlinearities as $g(x)=\frac{1}{x^{\alpha}}$ with $\alpha>0$, that appears when electrostatic or gravitational forces are considered. The existence of periodic solutions of this class of singular equations was started in [4] for the undamped case and was continued in [3] for the Liénard equation.

In this paper we prove that the existence of a bounded solution implies the existence of a unique $T$-periodic solution that attracts all bounded solutions. Besides, the set of initial conditions corresponding to bounded solutions is described as the graph of a decreasing function.

These results are related to a recent paper [5], where the authors prove similar results for equations with singular nonlinearities. However, the functions $f$ and $g$ were assumed to be more smooth and $f$ had to satisfy a quantitative estimate, namely,

$$
0<m \leq f(x) \leq M
$$

Received by the editors August 31, 1997.

1991 Mathematics Subject Classification. Primary 34C25, 54H20.

This work was supported by D.G.E.S. PB95-1203, M.E.C., Spain, and E.E.C. project ERBCHRX-CT94-0555. 
where $m, M$ are constants such that

$$
M \leq \frac{2+\sqrt{2}}{2-\sqrt{2}} m
$$

This condition was required in order to use Massera's convergence theorem due to R. A. Smith (see [7]).

The novelty of the present paper is the use of topological tools, such as free homeomorphisms (see [1]), together with truncation arguments, that allow us to remove not only the smoothness condition, but also the condition (1.2) on the friction term $f$, leaving only the non-negativity, a very reasonable hypothesis from the point of view of Mechanics.

Now, we are going to describe the structure of the paper. In Section 2 we study the monotonicity properties of equation (1.1) that will help us in the proof of the main results. In Section 3 we prove a convergence result by using the theory of free homeomorphisms together with a truncated argument. In Section 4 we prove a description of the set of bounded solutions. The situation recalls the second section, but in this case some technicalities are necessary when we go from the truncated case to the general one.

\section{COMPARISON OF SOlutions}

The following theorem is a key tool that we are going to use several times. Denote by $x\left(t ; t_{0}, x_{0}, v_{0}\right)$ the unique solution of the Cauchy problem with $\left(w^{-}, w^{+}\right)$ its maximal interval of definition.

Theorem 2.1. Let $x(t)=x\left(t ; t_{0}, x_{0}, v_{0}\right), y(t)=x\left(t ; t_{0}, x_{1}, v_{1}\right)$ be different solutions of (1.1) such that $x_{0} \leq x_{1}$ and $v_{0} \leq v_{1}$. Then,

$$
x(t)<y(t), \quad \forall t_{0}<t<\min _{i=0,1} w^{+}\left(t_{0}, x_{i}, v_{i}\right) .
$$

Proof. If the conclusion fails to hold, then there must be a first number $t_{1}$ after $t_{0}$ such that $x\left(t_{1}\right)=y\left(t_{1}\right)$. Set $z(t)=y(t)-x(t)$. Then $z(t)>0, t \in\left(t_{0}, t_{1}\right)$ and $z\left(t_{1}\right)=0$. Subtracting the respective equations and using the monotony of the function $g$,

$$
z^{\prime \prime}(t)+f(y(t)) y^{\prime}(t)-f(x(t)) x^{\prime}(t)>0, \forall t \in\left(t_{0}, t_{1}\right),
$$

now an integration over $\left(t_{0}, t_{1}\right)$ gives

$$
z^{\prime}\left(t_{1}\right)-v_{1}+v_{0}+\int_{x_{1}}^{y\left(t_{1}\right)} f(s) d s-\int_{x_{0}}^{x\left(t_{1}\right)} f(s) d s>0 .
$$

Thus, $z^{\prime}\left(t_{1}\right)>v_{1}-v_{0}-\int_{x_{1}}^{y\left(t_{1}\right)} f(s) d s+\int_{x_{0}}^{x\left(t_{1}\right)} f(s) d s=v_{1}-v_{0}+\int_{x_{0}}^{x_{1}} f(s) d s \geq 0$, but this is not possible.

Remark 2.2. This theorem does not imply that the Poincaré map is monotone with respect to the usual order in $\mathbb{R}^{2}$.

This Theorem has two consequences that we emphasize.

Corollary 2.3. Any couple of different solutions of (1.1) has at most one point in common, i.e. there exists at most a time $t^{*}$ such that

$$
x\left(t^{*}\right)=y\left(t^{*}\right)
$$

where $x(t)$ and $y(t)$ are solutions of (1.1). 
The proof is trivial.

Corollary 2.4. There exists at most one T-periodic solution of (1.1).

Proof. Suppose, by contradiction, that $x_{1}$ and $x_{2}$ are two different $T$-periodic solutions of (1.1). By the last corollary $x_{1}(t) \neq x_{2}(t)$ for all $t \in[0, T]$ and we can assume that $x_{1}(t)<x_{2}(t)$ for all $t \in[0, T]$. If we subtract the respective equations for $x_{1}$ and $x_{2}$, and use that $g$ is strictly decreasing, then

$$
x_{1}^{\prime \prime}(t)-x_{2}^{\prime \prime}(t)+f\left(x_{1}(t)\right) x_{1}^{\prime}(t)-f\left(x_{2}(t)\right) x_{2}^{\prime}(t)>0, \quad \forall t \in[0, T]
$$

and now we can integrate over $[0, T]$ obtaining a contradiction.

\section{A CONVERGEnCE RESUlt}

In accordance with the physical interpretation of $t$ as the time variable, we state the following definition.

Definition 3.1. A given function $x:\left(w^{-},+\infty\right) \rightarrow \mathbb{R}$ is said to be bounded in the future if there exist $r, s$ and $t_{0}$ such that

$$
a<r \leq x(t) \leq s<b, \quad \forall t>t_{0}
$$

The following theorem is the main result of this section.

Theorem 3.2. If there exists a solution that is bounded in the future, then there exists exactly one T-periodic solution. Moreover, every bounded solution in the future tends to the T-periodic one.

The proof of this theorem will require some previous results. The index considered in the next lemma is the Brouwer index.

Lemma 3.3. Let $P: \mathbb{R}^{2} \rightarrow \mathbb{R}^{2}$ be an orientation preserving homeomorphism with a unique fixed point $\left(x_{T}, v_{T}\right)$ such that

$$
\text { index }\left\{I-P,\left(x_{T}, v_{T}\right)\right\} \neq 1 \text {, }
$$

where I means the identity function. Then, for any $\left(x_{0}, v_{0}\right) \in \mathbb{R}^{2}$, one of these possibilities holds:

i) $P^{n}\left(x_{0}, v_{0}\right) \rightarrow\left(x_{T}, v_{T}\right)$ as $n \rightarrow+\infty$,

ii) $\left\|P^{n}\left(x_{0}, v_{0}\right)\right\| \rightarrow+\infty$ as $n \rightarrow+\infty$.

The proof of this lemma is a direct application of the results in [1]: Theorem 5.7 (of course of [1]) is applied in order to prove that $P$ is free in the sense exposed there. Now, we extend $P$ to a free homeomorphism between Riemann spheres, with $P(\infty)=\infty$. Now, we can apply Lemma 3.4 of the cited paper and the Omega limit set has to be a connected subset of the fixed point set. Then, the Omega limit set has to be a singleton, $\left(x_{T}, v_{T}\right)$ or the infinite point.

Remark 3.4. Theorem 5.7 of [1] has been used several times in the literature in order to obtain trivial dynamics (see [2] for a definition of trivial dynamics); it was used in [6] (Remark 2 after Theorem 5.1) and in [2] (Remark 3 after Theorem 2.1).

Lemma 3.5. Let us suppose that $a=-\infty, b=+\infty, f$ bounded, $g(-\infty)=+\infty$, $g(+\infty)=-\infty$ and there exists two constants $c, d>0$ such that

$$
|g(x)| \leq c+d|x|, \quad \forall x \in \mathbb{R} .
$$

With these assumptions, if $x(t)$ is a solution of (1.1) bounded in the future, then the derivative $x^{\prime}(t)$ is also bounded in the future. 
Proof. Since $x$ is bounded in the future, we can find $r, s, t^{*}$ such that

$$
r \leq x(t) \leq s, \quad \forall t \geq t^{*} .
$$

Now we can take $n_{0}$ such that $n_{0} T>t^{*}$ and applying the Mean Value Theorem in the interval $(n T,(n+1) T)$ with $n \geq n_{0}$, we obtain $t_{n} \in(n T,(n+1) T)$ such that

$$
x^{\prime}\left(t_{n}\right)=\frac{x((n+1) T)-x(n T)}{T},
$$

and then,

$$
\left|x^{\prime}\left(t_{n}\right)\right| \leq \frac{s-r}{T}
$$

for all $n \geq n_{0}$. Let's see that $x^{\prime}(t)$ is bounded in the interval $\left[n_{0} T,+\infty\right)$. Choose $t \geq n_{0} T$ and $n_{1}$ such that $n_{1} T \leq t \leq\left(n_{1}+1\right) T$. Then,

$$
x(t)=x\left(t ; t_{n_{1}}, x\left(t_{n_{1}}\right), x^{\prime}\left(t_{n_{1}}\right)\right),
$$

and since the equation is periodic,

$$
x(t)=x\left(t-n_{1} T ; t_{n_{1}}-n_{1} T, x\left(t_{n_{1}}\right), x^{\prime}\left(t_{n_{1}}\right)\right),
$$

also

$$
x^{\prime}(t)=x^{\prime}\left(t-n_{1} T ; t_{n_{1}}-n_{1} T, x\left(t_{n_{1}}\right), x^{\prime}\left(t_{n_{1}}\right)\right),
$$

then

$$
x^{\prime}(t) \in\left\{x^{\prime}\left(s ; s_{0}, x_{0}, v_{0}\right): s \in[0, T], s_{0} \in[0, T], x_{0} \in[r, s], v_{0} \in\left[\frac{r-s}{T}, \frac{s-r}{T}\right]\right\}
$$

which is a compact set that does not depend on $n_{1}$.

Proposition 3.6. In the assumptions of Lemma 3.5, every bounded solution in the future tends to the T-periodic solution.

Proof. We verify the assumptions of Lemma 3.3 by considering $P$ to be the Poincaré map. The conditions on $f$ and $g$ imply that $P$ is an orientation preserving homeomorphism from $\mathbb{R}^{2}$ onto $\mathbb{R}^{2}$.

We prove that there exists a unique fixed point with index -1 . Take $\alpha<\beta$ such that $g(\alpha)>p(t)>g(\beta)$ for all $t$. Then, $x_{1}(t) \equiv \alpha$ and $x_{2}(t) \equiv \beta$ is a couple of ordered strict lower and upper solutions, and it is a well known result (see for instance Proposition 2.1 in [8]) that the method of upper and lower solutions provides a $T$-periodic solution with index -1 , and by Corollary 2.4 this solution is unique. Now, the thesis of Lemma 3.3 implies that every solution with $|x(t)|+\left|x^{\prime}(t)\right|$ bounded in the future tends to the periodic one, and finally, Lemma 3.5 ends the proof.

Proof of Theorem 3.2. Let $x(t)$ be a solution bounded in the future, and let $r, s$ and $t^{*}$ be three numbers such that

$$
r \leq x(t) \leq s \quad t \geq t^{*} .
$$

Let $\hat{f}$ and $\hat{g}$ be extensions of $\left.f\right|_{[r, s]}$ and $\left.g\right|_{[r, s]}$ verifying the hypotheses of Lemma 3.5. Then, $x(t)$ is a solution of the differential equation

$$
x^{\prime \prime}+\hat{f}(x) x^{\prime}+\hat{g}(x)=p(t),
$$

in $\left[t^{*},+\infty\right)$, so by Proposition $3.6 x(t)$ tends to a $T$-periodic solution $x_{T}(t)$ of the differential equation (3.2), but using (3.1) we have that $x_{T}(t)$ verifies $r \leq x_{T}(t) \leq s$ for every $t \in \mathbb{R}$, and therefore $x_{T}$ is a $T$-periodic solution of (1.1). 
In conclusion, equation (1.1) has a $T$-periodic solution and $x$ tends to it. Now, by Corollary 2.4 the $T$-periodic solution is unique, and we conclude the proof.

\section{The SET OF BOUnded SOLUTiOns}

In this section, we are going to describe the geometry of the set of initial conditions of the bounded solutions in the future. By Theorem 3.2 of the last section, we know that this is the set of initial conditions of the global stable "manifold" of the periodic solution. Therefore, we can define

$$
W_{s}^{t_{0}}:=\left\{(x, v): x\left(t ; t_{0}, x, v\right) \text { is bounded in the future }\right\} .
$$

The existence of the stable manifold locally near the $T$-periodic solution can be deduced from general results on hyperbolic fixed points, since it is easy to prove that the periodic solution is hyperbolic when the coefficients are smooth (also $p$ has to be nonconstant). Note that here we do not need smoothness on the coefficients.

Proposition 4.1. Consider $\left(x_{1}, v_{1}\right),\left(x_{2}, v_{2}\right) \in W_{s}^{t_{0}}$ with $v_{1}<v_{2}$. Then, $x_{2}<x_{1}$.

Proof. Define $x_{1}(t)=x\left(t ; t_{0}, x_{1}, v_{1}\right)$ and $x_{2}(t)=x\left(t ; t_{0}, x_{2}, v_{2}\right)$. If the conclusion fails to hold, by using Theorem 2.1 we have that $x_{1}(t)<x_{2}(t)$ for all $t>t_{0}$. Moreover, by Theorem 3.2 both of the solutions tend to the periodic one, so $\lim _{t \rightarrow+\infty} x_{2}(t)-x_{1}(t)=0$ and $\lim _{t \rightarrow+\infty} x_{1}^{\prime}(t)-x_{2}^{\prime}(t)=0$.

Now, subtracting the respective equations as in Theorem 2.1 and integrating over $\left(t_{0}, t\right)$, we obtain that

$$
x_{2}^{\prime}(t)-x_{1}^{\prime}(t)-\left(v_{2}-v_{1}\right)+\int_{x_{2}}^{x_{2}(t)} f(s) d s-\int_{x_{1}}^{x_{1}(t)} f(s) d s>0 .
$$

Taking limits when $t$ tends to infinity and having in mind that

$$
\int_{x_{2}}^{x_{2}(t)} f(s) d s-\int_{x_{1}}^{x_{1}(t)} f(s) d s=\int_{x_{1}(t)}^{x_{2}(t)} f(s) d s-\int_{x_{1}}^{x_{2}} f(s) d s,
$$

we obtain that

$$
-\left(v_{2}-v_{1}\right)-\int_{x_{1}}^{x_{2}} f(s) d s \geq 0
$$

which is a contradiction.

Remark 4.2. Since $f$ can be unbounded is not so trivial that

$$
\int_{x_{1}(t)}^{x_{2}(t)} f(s) d s
$$

tends to zero, but this problem can be overcome if we observe that $f$ is bounded in a neighborhood of the range of the periodic solution (the set $\left\{x_{T}(t) / t \in[0, T]\right\}$ ).

An immediate consequence of this proposition is the following.

Corollary 4.3. Any couple of different solutions of (1.1) bounded in the future has no points in common, that is, $x_{1}(t) \neq x_{2}(t)$ for every time $t$ where both solutions are defined.

Now, the set $W_{s}^{t_{0}}$ is described in a special case. 
Lemma 4.4. Suppose the conditions of Lemma 3.5 hold. Then, there exist $-\infty \leq$ $\tilde{a}<\tilde{b} \leq+\infty$ and a continuous decreasing function $\varphi:(\tilde{a}, \tilde{b}) \rightarrow \mathbb{R}$ such that

$$
W_{s}^{t_{0}}=\{(x, \varphi(x)): x \in(\tilde{a}, \tilde{b})\}
$$

Proof. Define

$$
D^{+}=\left\{\left(x_{0}, v_{0}\right): \lim _{t \rightarrow+\infty} x\left(t ; t_{0}, x_{0}, v_{0}\right)=+\infty\right\}
$$

and

$$
D^{-}=\left\{\left(x_{0}, v_{0}\right): \lim _{t \rightarrow+\infty} x\left(t ; t_{0}, x_{0}, v_{0}\right)=-\infty\right\} .
$$

By using Theorem 2.1, $D^{+}$has the following property:

$\left(P_{1}\right)$ "If $(x, v) \in D^{+}$and $\tilde{x} \geq x, \tilde{v} \geq v$, then $(\tilde{x}, \tilde{v}) \in D^{+}$."

As a consequence $D^{+}$is a connected set of $\mathbb{R}^{2}$. Also, the symmetrical property holds for $D^{-}$:

$\left(P_{2}\right)$ "If $(x, v) \in D^{-}$and $\tilde{x} \leq x, \tilde{v} \leq v$, then $(\tilde{x}, \tilde{v}) \in D^{-}$"

so $D^{-}$is also connected. In this setting, we have the following:

Claim. $D^{+}$and $D^{-}$are open sets and $\mathbb{R}^{2}=D^{+} \cup D^{-} \cup W_{s}^{t_{0}}$.

The proof of this Claim will be done at the end. By using the Claim and properties $\left(P_{1}\right)$ and $\left(P_{2}\right)$, it is easy to prove that

$$
\tilde{I}=\left\{x \in \mathbb{R}: \exists v^{-}<v^{+} \text {such that }\left(x, v^{+}\right) \in D^{+},\left(x, v^{-}\right) \in D^{-}\right\}
$$

is an open interval $(\tilde{a}, \tilde{b})$ with $-\infty \leq \tilde{a}<\tilde{b} \leq+\infty$.

Then, for each $x \in \tilde{I}$ there exists $v$ such that $(x, v) \in W_{s}^{t_{0}}$, and by Corollary 4.3 this $v$ is unique. We define $\varphi(x):=v$ (see Figure 1 ).

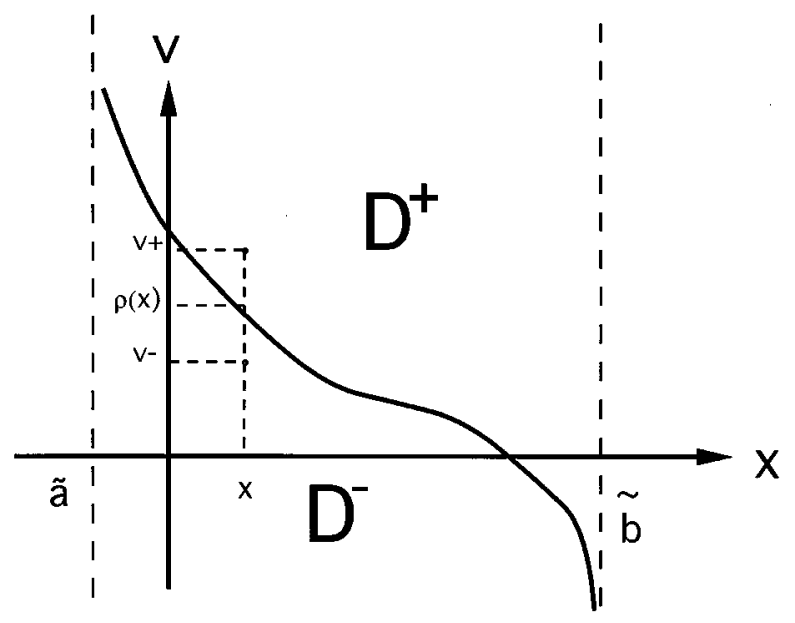

Figure 1

By construction,

$$
\{(x, \varphi(x)): x \in \tilde{I}\} \subset W_{s}^{t_{0}} .
$$

Now, we prove the remaining inclusion. Let us take $(x, v) \in W_{s}^{t_{0}}$; by the Claim and taking into account Proposition 4.1 we have that $(x, \tilde{v}) \in D^{+} \cup D^{-}$for every 
$\tilde{v} \neq v$. Moreover, by properties $\left(P_{1}\right)$ and $\left(P_{2}\right),(x, \tilde{v}) \in D^{+}$if $\tilde{v}>v$ and $(x, \tilde{v}) \in D^{-}$ if $\tilde{v}<v$. Consequently, $x \in \tilde{I}$ and $v=\varphi(x)$.

Therefore, we have proved the existence of a function $\varphi:(\tilde{a}, \tilde{b}) \rightarrow \mathbb{R}$ such that its graph is $W_{s}^{t_{0}}$. This function is decreasing by Proposition 4.1 and its graph $W_{s}^{t_{0}}=\mathbb{R}^{\not \models}-D^{+} \cup D^{-}$is closed, so this implies the continuity of $\varphi$.

Proof of the Claim. The hypotheses of Lemma 3.5 imply the existence of a couple of ordered numbers $\alpha<\beta$ such that

$$
g(\alpha)>p(t)>g(\beta), \quad \forall t \in[0, T] .
$$

By using the monotonicity of $g$ it is verified without difficulty that, if $x(t)>\beta$ and $x^{\prime}(t)=0$, then $x^{\prime \prime}(t)>0$, so every solution of (1.1) has no local maximum greater than $\beta$. Similarly, every solution of (1.1) has no local minimum smaller than $\alpha$. In particular, unbounded solutions in the future are not oscillatory. We will refer to these two properties as the non-oscillating property.

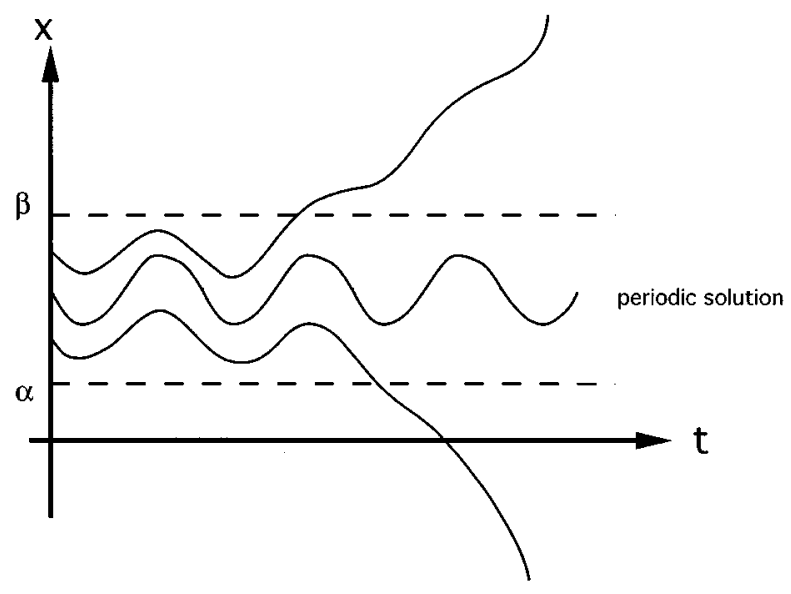

FiguRE 2. Region of oscillations

First, we are going to prove that $\mathbb{R}^{2}=D^{+} \cup D^{-} \cup W_{s}^{t_{0}}$. If $\left(x_{0}, v_{0}\right) \in \mathbb{R}^{2}$ does not belong to $W_{s}^{t_{0}}$, then by Theorem 3.2, the corresponding solution is unbounded. By the non-oscillating property stated above, it follows that $\left(x_{0}, v_{0}\right) \in D^{+} \cup D^{-}$.

Finally, let us prove that $D^{+}$is an open set (the proof for $D^{-}$is analogous). Let $\left(x_{0}, v_{0}\right) \in D^{+}$. It is possible to take $t_{1}$ such that $x\left(t_{1} ; t_{0}, x_{0}, v_{0}\right)>\beta$ and $x^{\prime}\left(t_{1} ; t_{0}, x_{0}, v_{0}\right)>0$. By continuous dependence, there exists an open ball $B_{R}\left(x_{0}, v_{0}\right)$ such that $x\left(t_{1} ; t_{0}, x_{1}, v_{1}\right)>\beta$ and $x^{\prime}\left(t_{1} ; t_{0}, x_{1}, v_{1}\right)>0$ for all $\left(x_{1}, v_{1}\right) \in B_{R}\left(x_{0}, v_{0}\right)$. Now the non-oscillating property together with Theorem 3.2 assure that

$$
\lim _{t \rightarrow+\infty} x\left(t ; t_{0}, x_{1}, v_{1}\right)=+\infty .
$$

Therefore $B_{R}\left(x_{0}, v_{0}\right) \subset D^{+}$and $D^{+}$is open.

Remark 4.5. From the argument used in Proposition 4.1, we deduce that if $f(x)>0$ for all $x \in \mathbb{R}$, the function $\varphi$ is strictly decreasing. Also, it is deduced from the proof that in this particular case we have a complete description of the asymptotic behavior of the solutions. 
Now, a truncation argument is used in order to get the same result in the general case. We consider the general case of the Liénard equation (1.1).

Theorem 4.6. Suppose the conditions for existence of a periodic solution. Then, there exist a non-empty open interval $\mathrm{I} \subset(a, b)$ and a continuous decreasing function $\varphi: \mathrm{I} \rightarrow \mathbb{R}$ such that

$$
W_{s}^{t_{0}}=\{(x, \varphi(x)): x \in \mathrm{I}\} .
$$

Proof. As usual, let $x_{T}(t)$ be the $T$-periodic solution, take $(r, s)$ satisfying

$$
r<x_{T}(t)<s, \forall t \in[0, T],
$$

and define

$$
W_{s}^{t_{0}}(r, s)=\left\{(x, v) \in W_{s}^{t_{0}} / r<x\left(t ; t_{0}, x, v\right)<s, \forall t \geq t_{0}\right\} .
$$

It is clear from (4.1) that this set is not empty.

The next Lemma contains essentially the proof of the Theorem.

Lemma 4.7. There exist $\tilde{I}$ an open subinterval of $(a, b)$ and $\psi: \tilde{I} \rightarrow \mathbb{R}$ a continuous and decreasing function, such that

$$
W_{s}^{t_{0}}(r, s)=\{(x, \psi(x)) / x \in \tilde{I}\}
$$

With this lemma the proof of the theorem follows easily. We take two sequences $r_{n} \rightarrow a$ and $s_{n} \rightarrow b, a<r_{n}<s_{n}<b$ such that (4.1) is satisfied for $\left(r_{n}, s_{n}\right)$ and we apply this Lemma to obtain $\psi_{n}: \tilde{I}_{n} \rightarrow \mathbb{R}$, such that

$$
W_{s}^{t_{0}}\left(r_{n}, s_{n}\right)=\left\{\left(x, \psi_{n}(x)\right) / x \in \tilde{I}_{n}\right\} .
$$

Since

$$
W_{s}^{t_{0}}=\bigcup_{n=1}^{\infty} W_{s}^{t_{0}}\left(r_{n}, s_{n}\right)
$$

the conclusion of the theorem holds noting that

$$
I=\bigcup_{n=1}^{\infty} \tilde{I}_{n}
$$

and

$$
\psi(x)=\psi_{n}(x)
$$

where $x \in \tilde{I}_{n}$. Only remains to prove Lemma 4.7 .

Proof of Lemma 4.7. First note that $W_{s}^{t_{0}}(r, s)$ only depends on the values of $f$ and $g$ over the compact set $[r, s]$, then we can take two extensions $\hat{f}$ and $\hat{g}$ of $f_{\mid[r, s]}$ and $g_{\mid[r, s]}$ satisfying the hypotheses of Lemma 3.5 and apply Lemma 4.4 to obtain a function $\hat{\varphi}:(\hat{a}, \hat{b}) \rightarrow \mathbb{R}$ such that $\hat{W}_{s}^{t_{0}}$ (the set of bounded solutions in the future for the modified equation (3.2)) has the expression

$$
\hat{W}_{s}^{t_{0}}=\{(x, \hat{\varphi}(x)), x \in(\hat{a}, \hat{b})\} .
$$

By Corollary 4.3, the set of bounded solutions in the future has to be ordered, then

$$
\tilde{I}=\left\{x / r<\hat{x}\left(t ; t_{0}, x, v\right)<s, \forall t \geq t_{0}\right\}
$$


is an interval ( $\hat{x}$ means the general solution for the modified equation (3.2)). Note that if we prove that the interval $\tilde{I}$ is open, then

$$
W_{s}^{t_{0}}(r, s)=\{(x, \hat{\varphi}(x)) / x \in \tilde{I}\} .
$$

So we will finish the proof if we prove that $\tilde{I}$ is open.

Take $x \in \tilde{I}$ and $\hat{a}<x_{1}<x<x_{2}<\hat{b}$. By construction we can find a number $t^{*}$ such that for $t \geq t^{*}$

$$
r<\hat{x}\left(t ; t_{0}, x_{1}, \hat{\varphi}\left(x_{1}\right)\right)<\hat{x}\left(t ; t_{0}, x_{2}, \hat{\varphi}\left(x_{2}\right)\right)<s ;
$$

then by Corollary 4.3 the solutions bounded in the future are ordered, so for all $y \in\left(x_{1}, x_{2}\right)$ and $t \geq t^{*}$

$$
r<\hat{x}\left(t ; t_{0}, y, \hat{\varphi}(y)\right)<s .
$$

Now, if $|y-x|$ is small, then $|\hat{\varphi}(y)-\hat{\varphi}(x)|$ is also, and we can apply continuous dependence to obtain $\varepsilon>0$ such that if $|y-x|<\varepsilon$ we have (4.2) for $t_{0} \leq t \leq t^{*}$.

Consequently, if $|y-x|<\varepsilon$ and $y \in\left(x_{1}, x_{2}\right)$ we have (4.2) for $t \geq t_{0}$ and, then $y \in \tilde{I}$. Therefore $\tilde{I}$ is open.

Remark 4.8. As before, if $f(x)>0$ for all $x \in \mathbb{R}$, the function $\varphi$ is strictly decreasing. Moreover, imposing additional restrictions on the nonlinearity $g$, it is possible to get a more complete picture of the dynamics of the equation, as is done in [5]. However, our interest is focused on the study of a general case, more than a detailed description in particular situations.

Remark 4.9. Minor modifications lead us to obtain similar results "in the past", that is, a description of the unstable manifold. In particular, the existence of a solution such that

$$
a<r \leq x(t) \leq s<b, \quad \forall t<t_{0}
$$

for some constants (this solution can be called bounded in the past) implies the existence of exactly one $T$-periodic solution. Also, every solution bounded in the past comes from the periodic one, i.e.

$$
\lim _{t \rightarrow-\infty}\left|x(t)-x_{T}(t)\right|+\left|x^{\prime}(t)-x_{T}^{\prime}(t)\right|=0,
$$

since there is some type of reversibility in the argument (the main tool was the freeness of the Poincaré map and $P$ is free if and only if $P^{-1}$ is, by definition).

Moreover, the set of initial conditions of the solutions bounded in the past can be described in a similar way as Theorem 4.6, only changing that $\varphi$ is in this case an increasing function.

Note that the change of variables $\tau=-t$ lead to a differential equation of the type (1.1) with negative friction term, so we can obtain the same results when the friction term does not change the sign. Nevertheless, in the case of negative friction term this equation does not seem to have physical meaning.

\section{ACKNOWLEDGMENT}

We thank Pedro Martínez Amores and Rafael Ortega for their useful comments about a first version of this paper. Also, we thank Rafael Ortega for encouraging us to work in this line. 


\section{REFERENCES}

[1] M. Brown, Homeomorphisms of two-dimensional manifolds, Houston J. of Math., 11, (1985), 455-469. MR 87g:57020

[2] J. Campos; R. Ortega; A. Tineo, Homeomorphisms of the disk with trivial dynamics and extinction of competitive systems, J. Diff. Equ. 138, (1997), 157-170. CMP 97:15

[3] P. Habets; L. Sanchez, Periodic solutions of some Liénard equations with singularities, Proc. Amer. Math. Soc., 109, (1990), 1035-1044. MR 90k:34049

[4] A.C. Lazer; S. Solimini, On periodic solutions of nonlinear differential equations with singularities, Proc. Amer. Math. Soc., 99, (1987), 109-114. MR 87k:34064

[5] P. Martínez-Amores; P. J. Torres, Dynamics of a periodic differential equation with a singular nonlinearity of attractive type, J. Math. Anal. and Appl., 202, (1996), 1027-1039. MR 97f:34027

[6] R. Ortega, Periodic Solutions of a Newtonian Equation: Stability by the Third Approximation, J. Diff. Equ., 128, (1996), 491-518. MR 97m:34077

[7] R.A. Smith, Massera's convergence theorem for periodic nonlinear differential equations, $J$. Math. Anal. and Appl., 120, (1986), 679-708. MR 87k:34068

[8] A. Zitan; R. Ortega, Existence of asymptotically stable periodic solutions of a forced equation of Liénard type, Nonlinear Anal., 22, (1994), 993-1003. MR 95f:34047

Departamento de Matemática Aplicada, Universidad de Granada, 18071 Granada, SPAIN

E-mail address: jcampos@goliat.ugr.es

E-mail address: ptorres@goliat.ugr.es 